\title{
Self-management education for adults with poorly controlled epilepsy (SMILE (UK)): statistical, economic and qualitative analysis plan for a randomised controlled trial
}

\author{
Nicholas Magill ${ }^{*}$, Leone Ridsdale², Laura H. Goldstein ${ }^{3}$, Paul McCrone $^{4}$, Myfanwy Morgan $^{5}$, Adam J. Noble ${ }^{6}$,
} Gus Baker ${ }^{7}$, Mark Richardson², Stephanie Taylor ${ }^{8}$ and Sabine Landau ${ }^{1}$

\begin{abstract}
Background: There is a need to test the effectiveness of new educational interventions for people with poorly controlled epilepsy. The SMILE (self-management education for adults with poorly controlled epilepsy) trial evaluates a complex service intervention that involves a 2-day self-management course with the aim of improving quality of life and clinical outcomes. This article describes the statistical, economic, and qualitative analysis plan for the trial.
\end{abstract}

Methods and design: SMILE is a pragmatic, parallel design, two-arm, multi-centre randomised controlled superiority trial of a group-based interactive course compared with treatment as usual for people who have experienced two or more seizures in the past 12 months.

Results: A summary of the objectives and design of the trial are reported as well as the manner in which the data will be summarised and inferentially analysed. This includes the type of modelling that will be employed for each of the primary and secondary outcomes and the methods by which the assumptions of these models will be checked. Strategies are described for handling clustering of outcome data, missing observations, and treatment non-compliance.

Conclusion: This update to the previously published trial protocol provides a description of the trial analysis which is transparent and specified before any outcome data are available. It also provides guidance to those planning the analysis of similar trials.

Trial registration: Current Controlled Trials ISRCTN57937389; date assigned: 27 March 2013.

Keywords: Statistical analysis plan, SMILE trial, epilepsy, self-management education, randomised controlled trial

\section{Update}

Background

Epilepsy

Epilepsy is a long-term neurological condition, which affects approximately $1 \%$ of the UK population [1]. After diagnosis, roughly $40 \%$ of patients continue to experience at least two seizures per year [2] and the consequences for this group include elevated risks of injury,

\footnotetext{
* Correspondence: nicholas.magill@kcl.ac.uk

'Department of Biostatistics, Institute of Psychiatry, Psychology and Neuroscience, King's College London, PO 20, Denmark Hill Campus, 16 De Crespigny Park, London SE5 8AF, UK

Full list of author information is available at the end of the article
}

depression, and premature death [1]. From the perspective of health service provision, poorly controlled epilepsy is expensive $(€ 2,000-€ 11,500$ per case in 2004) [3]. The overwhelming majority of admissions for epilepsy are on an emergency basis [4], making it the sixth most common cause of emergency admission for chronic conditions in the UK [5] and adding considerably to healthcare cost.

Providing patients with the ability to manage longterm conditions and reducing emergency admissions are key aims of the National Health Service (NHS). For people with poorly controlled epilepsy, this means increasing patients' confidence in their ability to recognise 
triggers for seizures [6] and improving their adherence to antiepileptic drugs (AEDs) [7, 8]. Routine NHS policy for another chronic illness, diabetes, is the offer of group education (e.g., diabetes education and selfmanagement for ongoing and newly diagnosed, or DESMOND [9]), and there is now demand for similar courses for those with epilepsy. This is supported by the finding that a third of those with poorly controlled epilepsy have been told little about the disease and the side effects of AEDs [10].

Of a number of epilepsy interventions reviewed by the Cochrane collaboration $[11,12]$, only one has been thoroughly assessed and shown potential benefit in the UK. This group intervention was developed in Germany, where it was called Modular Service Package in Epilepsy (MOSES) [13]. A trial of this reported improved knowledge about the condition, enhanced control of seizures, and better acceptance of AEDs. The intervention has been adapted for use in the UK and is referred to here as the SMILE (self-management education for adults with poorly controlled epilepsy) trial.

\section{SMILE trial}

The SMILE trial is a parallel design, two-arm, multicentre randomised controlled superiority trial of a 2-day self-management course for adults with poorly controlled epilepsy. Participants are randomly allocated to receive either SMILE and treatment as usual (TAU) or TAU alone, and randomisation is stratified by treatment centre. The aim is to test whether the intervention affects quality of life, clinical outcomes, and costeffectiveness of health service use at 6 and 12 months after randomisation. Blinding is planned for outcome assessors and the trial statistician. See protocol article for further details [14].

This article describes the statistical analysis plan for the main analyses of the trial. The plan was finalised and approved by the Trial Steering Committee on 3 March 2014, before any outcome data were available.

\section{Research questions \\ Primary objective}

The primary objective is to examine the treatment difference in Quality of Life in Epilepsy-31 (QOLIE-31) [15] scores between participants allocated to self-management education (SMILE) versus those allocated to TAU at 12 months after randomisation.

\section{Secondary objectives}

The secondary objectives are the following:

1. To investigate the treatment difference in QOLIE-31 between participants allocated to SMILE and those allocated to TAU at 6 months after randomisation.
2. To investigate the treatment effect on seizure frequency as recorded using the scale of Baker et al. [16] between participants allocated to SMILE and those allocated to TAU at 6 and 12 months after randomisation.

3. To investigate the treatment effect on seizure frequency as recorded using the scale of Thapar et al. [17] between participants allocated to SMILE and those allocated to TAU at 6 and 12 months after randomisation.

4. To investigate the treatment effect on time elapsed since last seizure of allocation to SMILE compared with those offered TAU at 6 and 12 months after randomisation.

5. To investigate the treatment difference in selfperceived impact of epilepsy (as measured by the Impact of Epilepsy scale [18]) between those allocated to SMILE and those offered TAU at 6 and 12 months after randomisation.

6. To investigate the treatment difference in medication management as measured by the Epilepsy Self-Management Questionnaire [7] between participants allocated to SMILE and those allocated to TAU at 12 months after randomisation.

7. To investigate the treatment difference in medication adverse events (AEs) as measured by two questions from QOLIE-31 [15] between participants allocated to SMILE and those allocated to TAU at 6 and 12 months after randomisation.

8. To investigate the treatment effect on psychological distress as measured by the Hospital Anxiety and Depression Scale (HADS) [19] between participants allocated to SMILE and those allotted to TAU at 12 months after randomisation.

9. To investigate the treatment difference in perceived stigma as measured by Jacoby's Stigma of Epilepsy scale [20] between participants allocated to SMILE and those allotted to TAU at 12 months after randomisation.

10.To investigate the treatment effect on self-efficacy in management of epilepsy (using the mastery/control of epilepsy scale [21]) between participants allocated to SMILE and those allocated to TAU at 12 months after randomisation.

11. To investigate the treatment difference in costeffectiveness of health service use between participants randomly assigned to SMILE and those randomly assigned to TAU at 12 months after randomisation.

12.To conduct an in-depth qualitative interview study to investigate SMILE users' views on their participation in, and the perceived benefits of, the intervention.

See protocol for further details of measures [14]. 


\section{Trial design}

\section{Recruitment procedure and randomisation}

Research workers visit participants at their home or a place of their choice and explain the trial in detail. Those individuals who meet the eligibility criteria are asked to provide informed written consent and baseline assessments. Consenting individuals who provide baseline data are randomly assigned to one of the treatment arms in a 1:1 ratio. See Fig. 1 for an illustration of the process by which patients are screened, recruited, randomly assigned, and followed up. Randomisation is at the patient level and is performed by using an online randomisation system set up by the King's Clinical Trials Unit (KCTU) at the Institute of Psychiatry, Psychology and Neuroscience in London. Randomisation is stratified by treatment centre. A set of patients is randomly assigned with fixed block sizes of two to ensure that equal numbers of patients are allocated to the two arms within each treatment centre. The procedure is as follows: on receipt of the baseline questionnaire and after between 14 and 24 participants have been recruited to a site, the trial coordinator electronically submits details of the set of participants to the KCTU. The system immediately notifies the trial coordinator of successful randomisation and the trial arm to which the participant has been allocated. A record of the allocation outcome is kept in the KCTU randomisation database and later linked to the main dataset.

Ethical approval was given by the National Research Ethics Service London - Fulham (reference 12/LO/ 1962).

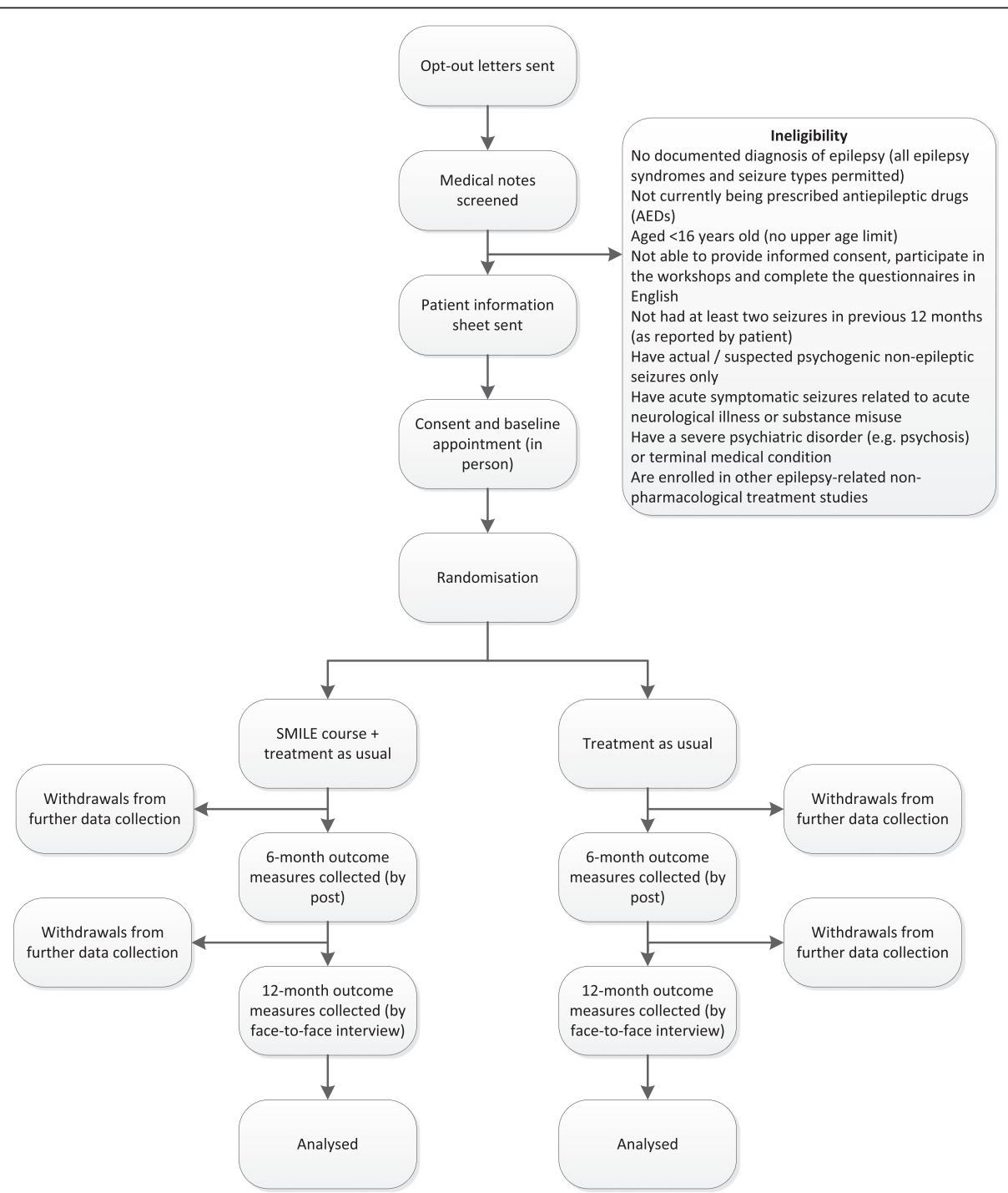

Fig. 1 CONSORT (Consolidated Standards of Reporting Trials) diagram. SMILE self-management education for adults with poorly controlled epilepsy 


\section{Sample size}

The primary intention-to-treat (ITT) analyses will compare two equal-sized treatment arms, treatment or control, on the quality-of-life scale (QOLIE-31) at 12 months. Two drug trials that used this scale as an outcome showed standardised effect sizes of $d=0.33$ [22] and 0.59 [23]. An overall sample size of 320 participants (randomly assigned 1:1) would provide $91.3 \%$ power to detect an effect size of $d=0.40$ on the QOLIE-31 using an analysis of covariance with two-sided $5 \%$ significance tests. A value of $d=0.40$ corresponds to a change of around 6-7 points on the overall quality-of-life score.

Since the active treatment is a group treatment delivered by different educational facilitators within sites, we must allow for standard error inflation due to training group effects. Assuming an average group size of 10 people with epilepsy and an intra-group correlation between QOLIE-31 scores of intra-class correlation coefficient (ICC) equal to 0.025 (a correlation of 0.05 would be expected for intermediate outcomes but would be lower for distal outcomes such as quality of life [24]), we estimated that 160 patients in the control arm and 16 groups of 10 patients in the SMILE arm would provide $91.3 \%$ power to detect an effect of $d=0.40$.

The estimated attrition rate was based on data from a study of rehabilitation for people with severe epilepsy which had a $25 \%$ loss at 1 year (NIHR SDO 08/1815/ 234). Therefore, to ensure adequate and equal-sized groups, a sample of 428 patients is required ( $n=320$ / 0.75 ; 214 patients per arm).

\section{Timing and visit windows of follow-up}

Participants will complete a limited selection of followup measures at 6 months after randomisation by using a questionnaire sent to them in the post. This comprises quality of life, seizure frequency, time elapsed since last seizure, and impact of epilepsy. The full range of followup measures will be completed at 12 months after randomisation by face-to-face interview with a research assistant. The aim is for follow-up assessments to be collected within 3 weeks of the intended date.

\section{Minimising attrition}

It is expected that some participants will be lost to follow-up and this has been accounted for in the sample size calculation. However, every effort will be made to minimise such attrition, by following the recommendations of recent research [25]. A sequence of telephone reminders will be used to maximise the collection of follow-up data, especially at the 6-month assessment. This assessment has been specifically condensed in order to reduce patient burden. An additional measure to increase adherence is the distribution of $£ 20$ vouchers following the 12-month assessment.

\section{Blinding}

Outcome assessors and the trial statistician will be blind to participants' allocated treatment arm. At the start of each follow-up assessment, interview participants are requested not to reveal to the researcher which group they were allocated to. If a researcher is unblinded at the 6month outcome visit, a different researcher will conduct the next follow-up assessment. Additionally, the trial databases have been designed specifically to enable data entry without unblinding researchers. This has been achieved by creating separate databases for recording outcome data, course attendance, and educational facilitator information. We are assessing how well researchers were kept blind by asking the assessor to make a guess as to which treatment each participant was allocated to at the end of follow-up.

\section{Reporting}

The trial results will be reported in a manner that is consistent with the recommendations of the updated CONSORT (Consolidated Standards of Reporting Trials) guidelines for parallel group trials [26]. Additionally, the report will follow the CONSORT extensions on pragmatic and non-pharmacologic trials [27, 28].

\section{Description of data \\ Baseline comparability of randomly assigned groups}

Baseline descriptions of participants' demographic and clinical data will be reported by treatment group and overall. Minima and maxima, means and standard deviations, and medians and quartiles will be used for continuous variables as appropriate. Frequencies and proportions will be presented for categorical variables. No significance testing will be used to test baseline differences between the trial arms.

\section{Adherence to allocated treatment}

Adherence is defined as being in attendance at the beginning and end of each of the two days of the selfmanagement course. Adherence versus non-adherence with the treatment will be described in terms of baseline variables. The reasons for withdrawal from treatment will be summarised. Treatment adherence will be described by using attendance and reasons for nonattendance.

\section{Loss to follow-up and missing data}

Withdrawal from trial follow-up (attrition) will be reported by intervention group. Moreover, the proportions of participants who are missing values on each variable will be summarised by trial arm and time point.

The baseline characteristics of those missing follow-up will be compared with those with complete follow-up. The relationship between baseline characteristics and 
missing data will also be investigated graphically. Factors affecting missingness will be examined by using logistic regression for a missingness variable. This will be done by generating a binary variable for missingness at 12 months after randomisation and regressing this on prerandomisation (baseline) variables. All outcome variables will be assessed before choosing a representative selection.

The relationship between compliance and loss to follow-up will be assessed. This will be done by using binary variables for completion of all training sessions and for drop-out at 12 months. The relationship between these variables will be tested by using a chisquared test. The results of these analyses will inform the need to use multiple imputation (MI) in the formal analysis. This is because these post-randomisation variables cannot be included as covariates in the model without changing the meaning of the results.

\section{Adverse events}

AEs, adverse reactions, serious AEs, and serious adverse reactions will be summarised by treatment arm.

\section{Descriptive statistics for outcome measures}

Each of the outcome measures will be described by treatment group and time point. Means and standard deviations or medians and interquartile ranges will be used for continuous variables; box plots, histograms, and Q-Q plots will be used to assess whether the distribution of a variable is normal. Frequencies and proportions will be used to describe categorical variables.

\section{Inference and modelling Inferential analysis}

The main statistical analyses will estimate the difference in mean outcomes between patients randomly assigned to SMILE and TAU versus TAU alone by ITT (i.e., treatment groups defined by result of randomisation rather than treatment received) at the various post-treatment observation time points. This will be done by using mixed-effects linear regression modelling for longitudinal data, including outcome measures at all time points and conditioning on the stratification variable (treatment centre) and baseline measures. Group difference estimates and associated confidence intervals will be reported.

Missing post-randomisation assessments will be dealt with by fitting adequate linear mixed models to all the variables by using maximum likelihood (ML) methods. Such an approach provides valid inferences under the assumption that the missing data mechanism is ignorable (missing at random, or MAR) and provided that predictors of missingness are included as covariates in the model.
Group difference estimates and associated $95 \%$ confidence intervals will be reported. The trial statistician will remain blind until the main analyses have been completed. Some analyses, such as modelling training group effects, estimating complier average causal effects (CACEs), and summarising numbers trained by each educational facilitator, cannot be performed blind. Such analyses will be carried out last in order to preserve blindness for as long as possible.

The significance level will be $5 \%$ (two-sided) for the primary outcome. Secondary analyses will be carried out at the $5 \%$ level but will have to be interpreted with care as multiple testing is not taken account of. Sensitivity analyses will be used to assess the robustness of conclusions to non-ignorable missing outcome data.

\section{Analysis of primary outcome}

The analysis population will include all randomly assigned patients. The primary outcome is quality of life, as measured by the QOLIE-31, at 12 months after randomisation. Quality of life at both post-treatment time points (6 and 12 months after randomisation) will be modelled simultaneously. These outcomes will constitute the dependent variable, and quality of life at baseline, treatment centre, baseline predictors of drop-out, trial arm, time dummy variables, and a treatment"time interaction term will be included as explanatory variables. This last term will allow the model to provide the treatment effect estimate at the primary time point (12 months after randomisation). The covariance matrix of the repeated measures will be carefully modelled: an unstructured covariance matrix and the covariance matrix implied by a participant-varying random intercept model will be formally compared, and the best covariance structure will be identified. This analysis is valid provided that missing values in quality-of-life outcomes are MAR. This is to say that, given the observed variables that have been included in the analysis model, the missingness pattern does not depend on any unobserved/ unmodelled data.

The relationship between baseline variables and missing outcome data will be assessed by using logistic regression with an outcome variable that represents whether outcome quality-of-life data are present or missing. Should any baseline variables be predictive of missingness, these will be included in the model as covariates. Should the post-treatment variable "compliance with treatment" predict missingness, MI will be used to approximate an ML estimator under this form of MAR [29]. The impact of departures from MAR on treatment effects will be assessed by using sensitivity analysis [30].

Potential clustering due to participants attending the same educational group (and therefore sharing the same interventionist) in the SMILE arm will also be assessed 
by exploring the variability between these groups. This will be summarised by using the ICC. It is expected that a statistical dependence will exist and that this will need to be addressed in the modelling process. Therefore, it is anticipated that models will need to include random effects that vary with educational group in this trial arm.

\section{Analysis of secondary outcomes}

Secondary patient outcomes relating to the quality of life (at 6 months after randomisation), impact of epilepsy, medication management, psychological distress, and mastery/control of epilepsy will be analysed by using linear mixed models and an approach similar to that described above.

Medication adverse effects and perceived stigma are unlikely to be normally distributed. Transformations will be investigated for these outcomes. Failing this, other types of mixed models, such as a mixed logistic model, will be considered.

Seizure frequency as measured by using the scale of Thapar et al. is a count variable and will be analysed by using a type of Poisson mixed model, and allowance will be made for overdispersion/varying exposure periods. Seizure frequency using the scale of Baker et al. is an ordinal variable and will be analysed by using a mixed ordinal logit model.

Time (days) elapsed since last seizure is measured prerandomisation (at baseline) and at 6 and 12 months after randomisation. Such a variable will be analysed after a $\log$ transformation. If the data are truncated, survival modelling will be considered.

\section{Statistical considerations}

\section{Stratification and clustering}

Randomisation is stratified by treatment centre (of which there are up to 15). Therefore, it is important to include this variable as a covariate in the modelling process. The structure of the majority of the data is longitudinal, and repeated measurements are taken at baseline and 6 and 12 months after randomisation. This correlation between participants' repeated observations is being taken into account by a modelling process for the covariance matrix. It is also possible that clustering will be seen within educational facilitators/educational groups within the SMILE arm.

\section{Missing scale items}

The number (percentage) with complete data will be reported. The ideal approach is to use missing value guidance provided by the developers of the scales. When such guidance is not available, scales will be prorated for an individual if not more than $20 \%$ of items are missing. For example, in a scale with 10 items, prorating will be applied to individuals with one or two items missing.
The average value for the eight or nine complete items will be calculated for that individual and used to replace the missing values. The scale score will be calculated on the basis of the complete values and these replacements.

\section{Missing baseline data}

Missing baseline data should not be a problem. However, if we encounter missing baseline values of outcome variables, these can be singly imputed according to White and Thompson [31] without incurring bias in trial arm effect estimates.

\section{Missing outcome data}

Where there are two outcome time points, missing postrandomisation assessments will be dealt with by fitting linear mixed models to all the available data by using maximum likelihood methods. Such an approach provides valid inferences under the assumptions that the missing data mechanism is ignorable (or MAR) and that pre-randomisation data are available for the scale. If post-treatment variables such as compliance with treatment are found to be predictive of drop-out, multiple imputation will be considered. This will be assessed through the use of a chi-squared test of independence.

\section{Method for handling multiple comparisons}

For the primary outcome, no formal adjustment of the $P$ value for multiple testing is necessary. The analyses of the secondary outcomes will also not be adjusted for multiple outcome comparisons. Thus, care should be taken in the interpretation of inferences for the numerous secondary outcomes. (Results will need to be interpreted as hypotheses-generating and subject to replication.)

\section{Method for handling non-compliance}

In addition to the primary ITT analysis, the effect of actually receiving treatment as defined in the protocol (its efficacy) will be estimated for the primary outcome. If non-adherence with the active treatment is high, a CACE-type analysis will be considered. Specifically, instrumental variable (IV) methods will be used to assess the efficacy of the SMILE treatment. Such methods evaluate the causal effect of SMILE on a clinical outcome in the subpopulation who would comply with SMILE. The application of IV methods for explanatory evaluation of randomised controlled trials has been advocated because random allocation itself provides a strong instrument for treatment receipt [32]. IV regression will be carried out by using the two-stage least squares estimator as implemented in the Stata command "ivregress 2sls". 


\section{Model assumption checks}

The models assume normally distributed outcomes; this will have been checked when describing the data, and if substantial departures from normality occur, transformations will be considered. Residuals will be plotted to check for normality and inspected for outliers. This is expected to be unnecessary for the primary outcome but will be undertaken partly in order to explore the data and to maintain a consistent method for all outcomes.

\section{Sensitivity analyses}

The impact of departures from MAR on treatment effects of continuous outcomes will be assessed by using sensitivity analysis. This will be done for the quality-oflife outcomes and will be based on the investigators' opinion about the range of possible mean differences in outcome between those with missing data and those with observed values in the two trial arms [30].

\section{Planned subgroup analyses}

The trial has not been designed (powered) to assess treatment effects within subgroups. As a consequence, no such subgroup analyses are described.

\section{Software}

Data management will employ an online data collection system for clinical trials (MACRO; InferMed Limited, London, UK). This is hosted on a dedicated server at King's College London and managed by the KCTU. The KCTU Data Manager will extract data periodically as needed. Stata will be used for data description and the main inferential analysis.

\section{Economic analysis plan}

\section{Heath economic objectives (secondary objective 11)}

We will take both a health service and a societal perspective in the economic evaluation. The costs of the intervention will be calculated by taking into account staff time needed for training, supervision, and delivery of the educational session and will also include overheads and capital costs. The cost per session will be estimated by combining the above information with activity data. The Client Service Receipt Inventory will be adapted and used to record the use of other services and also unpaid carer time and time lost from work. The service use data will be combined with relevant unit cost information [33]. Lost employment costs will be calculated by combining lost work time with average wage rates. Health-care and societal costs at follow-up will be compared between the two arms by using a regression model with baseline costs controlled for. Cost data are often skewed, and we will use bootstrapping to generate $95 \%$ confidence intervals around the cost differences. To assess cost-effectiveness, we will combine costs with data for the primary outcome measure at 12 months. Cost utility will be assessed by combining costs with quality-adjusted life years (QALYs), which will be generated from the European Quality of Life-5 Dimensions (EQ-5D) questionnaire. If the intervention results in better outcomes and lower costs, it will be considered to be "dominant". If it results in better outcomes and higher costs, incremental cost-effectiveness ratios will be calculated to show the extra cost incurred to achieve a oneunit improvement on the QOLIE-31 or one extra QALY (both at 12 months). Uncertainty around the estimates of cost-effectiveness and cost utility will be made by taking 1000 cost-outcome combinations at random (and with replacement) from the data by using bootstrap methods and plotted on a cost-effectiveness plane. Interpretation of the results will use cost-effectiveness acceptability curves to show the probability that the intervention is the most cost-effective option for a range of different values placed on an improvement in outcome. For QALYs, the range will be $£ 0$ to $£ 100,000$. The range for improvements on the QOLIE-31 will be chosen so that values at which the intervention or TAU has a $50 \%$ and $70 \%$ and $90 \%$ likelihood of being costeffective are identified.

\section{Economic measures}

Client Services Receipt Inventory: This will record contacts with health-care services at baseline and over the follow-up. It includes hospital admissions, contact with primary and community care, and receipt of care from family and friends. In addition, it includes lost work time.

EQ-5D: QALYs will be calculated from the EQ-5D health state classification instrument. This covers five domains: mobility, self-care, usual activities, pain/discomfort, and anxiety/depression. For each domain, the respondent chooses one of five levels of functioning, from good to poor. The five levels for each of the five domains are used to define unique health states to which a pre-estimated "utility" value will be attached.

\section{Qualitative analysis plan (secondary objective 12) \\ Aim}

Qualitative studies are increasingly conducted as a component of the evaluation of "complex" interventions with the aim of supplementing quantitative measures and contributing to the wider implementation of health-care interventions [34]. The present study aimed to complement the quantitative measures by providing an indepth account of (1) participants' perceptions of what they valued and any negative aspects of the intervention and (2) whether and in what ways participants continued to make use of the training received. 


\section{Methods}

\section{Study group}

Pilot interviews at 12 months post-intervention have indicated that participants were often not able to recall whether they had learned particular information about epilepsy and its management from the intervention or through other sources. This supports evidence that ongoing seizures do impair the laying down of memory [35]. However, reducing the period between intervention and interviews would have possible implications for subsequent outcome measurement. Therefore, we will interview the control group participants 2-6 months after they have received the control group intervention and have completed the three phases of quantitative data collection.

\section{Recruitment}

The study will be based on around 20-24 study participants who were randomly assigned to the SMILE (UK) control group, and the precise sample number will depend on when sampling saturation has been achieved and the condition that no new issues or explanations are elicited by further interviews [36]. Participants will be selected purposively to achieve a study group that is inclusive in terms of differences in gender, age, ethnicity, severity of seizures, and depression score.

\section{Interviews}

These will take place at participants' homes or a convenient public place if preferred. Interviews will be semistructured and conducted in a conversational manner with probes, facilitation, and clarification of responses as required. The topics covered will include participants' experiences in taking part in the courses, their perceptions of things they valued and found of particular benefit and any negative aspects as well as any factors that encouraged or hindered their participation in the courses and whether and in what ways they have continued to make use of the training. The content of the course workbook will also be discussed in relation to participants' own needs and lifestyles.

\section{Analysis}

The interviews will be audio-recorded (with consent) and transcribed. The analysis will proceed alongside data collection and will be based on a framework approach [37]. This is suitable for small numbers of cases and ensures that each case is fully taken into account in the analysis. This analytic approach requires identifying initial themes that are then grouped into main and subthemes. This is applied to the raw transcript data for each interview, and a thematic "chart" is created that summarises information for each theme and allows cross-case and within-case analysis through a process of constant comparison, and particular attention is paid to deviant cases. This approach to analysis can be undertaken manually or by using new framework matrices from NVivo9 or 10. Two members of the research team will participate in data analysis to reduce bias in the identification and interpretation of themes and categories.

\section{Outputs}

The findings of this nested qualitative study will contribute to outcome assessment of the trial by providing explanations and a greater understanding of the quantitative assessment as well as exploring issues not covered by these assessments. The findings may also be written up as a stand-alone article that examines patient-based assessments of a self-management intervention.

\section{Abbreviations}

AE: Adverse event; AED: Antiepileptic drug; CACE: Complier average causal effect; CONSORT: Consolidated Standards of Reporting Trials; EQ-

5D: European Quality of Life-5 Dimensions; ICC: Intra-class correlation coefficient; ITT: Intention to treat; IV: Instrumental variable; KCTU: King's Clinical Trials Unit; MAR: Missing at random; MI: Multiple imputation; ML: Maximum likelihood; NHS: National Health Service; QALY: Qualityadjusted life year; QOLIE-31: Quality of Life in Epilepsy-31 scale; SMILE: SelfManagement education for adults with poorly controlled epilepsy; TAU: Treatment as usual..

\section{Competing interests}

The authors received a contribution from Sanofi UK (Guilford, Surrey, UK) to enable publishing of the patient workbook. They declare that they have no other competing interests.

\section{Authors' contributions}

NM and SL drafted and finalised the statistical section of the analysis plan. PMcC wrote the health economics analysis plan. MM provided the qualitative analysis plan. LR (chief investigator), LHG, AJN, GB, MR and ST provided suggestions and comments on the manuscript. All authors read and approved the final version of the manuscript.

\section{Acknowledgements}

We would like to acknowledge members of our trial steering (Anne Rogers, Phillip Smith, Henry Smithson, Ajay Thapar, Barbara Penney and Howard Ring) and data monitoring (Gene Feder, Mark Manford and Adrian Mander) committees, who all gave useful contributions to the writing of the analysis plan.

This research is funded by the National Institute for Health Research (NIHR) Health Technology Assessment program (reference 09/165/01). This study is also supported by the United Kingdom Clinical Research Collaborationregistered King's Clinical Trials Unit at King's Health Partners, which is partly funded by the NIHR Biomedical Research Centre for Mental Health at South London and Maudsley NHS Foundation Trust and King's College London and the NIHR Evaluation, Trials and Studies Coordinating Centre.

LHG and SL receive salary support from the NIHR Dementia Biomedical Research Unit (LHG) and the NIHR Biomedical Research Centre for Mental Health (SL) at the South London and Maudsley NHS Foundation Trust and King's College London. The views expressed are those of the authors and not necessarily those of the NHS, the NIHR, or the Department of Health.

\section{Author details}

${ }^{1}$ Department of Biostatistics, Institute of Psychiatry, Psychology and Neuroscience, King's College London, PO 20, Denmark Hill Campus, 16 De Crespigny Park, London SE5 8AF, UK. ²Department of Basic and Clinical Neurosciences, Institute of Psychiatry, Psychology and Neuroscience, King's College London, PO 43, Denmark Hill Campus, 16 De Crespigny Park, London SE5 8AF, UK. ${ }^{3}$ Department of Psychology, Institute of Psychiatry, Psychology 
and Neuroscience, King's College London, PO 77, Denmark Hill Campus, 16 De Crespigny Park, London SE5 8AF, UK. ${ }^{4}$ Department of Health Service and Population Research, Institute of Psychiatry, Psychology and Neuroscience, King's College London, PO 24, Denmark Hill Campus, 16 De Crespigny Park, London SE5 8AF, UK. ${ }^{5}$ Division of Health and Social Care Research, School of Medicine, King's College London, 7th Floor Capital House, 42 Weston Street, London SE1 3QD, UK. ${ }^{6}$ Department of Psychological Sciences, Institute of Psychology, Health and Society, The Whelan Building, University of Liverpool, Brownlow Hill, Liverpool L69 3GB, UK. ${ }^{7}$ Department of Molecular and Clinical Pharmacology, University of Liverpool, Ashton Street, Liverpool L69 3GE, UK. ${ }^{8}$ Barts \& The London School of Medicine and Dentistry, Centre for Primary Care and Public Health, Blizard Institute, Abernethy Building, 2 Newark Street, London E1 2AT, UK.

Received: 13 January 2015 Accepted: 29 May 2015 Published online: 12 June 2015

\section{References}

1. Ridsdale L, Charlton J, Ashworth M, Richardson MP, Gulliford MC. Epilepsy mortality and risk factors for death in epilepsy: a population-based study. $\mathrm{Br}$ J Gen Pract. 2011;61:e271-8. doi:10.3399/bjgp11X572463.

2. Moran NF, Poole K, Bell G, Solomon J, Kendall S, McCarthy M, et al. Epilepsy in the United Kingdom: seizure frequency and severity, anti-epileptic drug utilization and impact on life in 1652 people with epilepsy. Seizure. 2004;13:425-33. doi:10.1016/j.seizure.2003.10.002.

3. Pugliatti $M$, Beghi E, Forsgren $L$, Ekman M, Sobocki P. Estimating the cost of epilepsy in Europe: a review with economic modeling. Epilepsia. 2007:48:2224-33. doi:10.1111/j.1528-1167.2007.01251.x.

4. Noble AJ, Goldstein LH, Seed P, Glucksman E, Ridsdale L. Characteristics of people with epilepsy who attend emergency departments: prospective study of metropolitan hospital attendees. Epilepsia. 2012;53:1820-8. doi:10.1111/j.1528-1167.2012.03586.x.

5. NHS Health and Social Care Information Centre (HSCIC) Clt. CCG Outcomes Indicator Set: Emergency Admissions. http://www.hscic.gov.uk/catalogue/ PUB10584/ccg-ind-toi-mar-13-v4.pdf, accessed in October 2013.

6. NCGC. The Epilepsies: The Diagnosis and Management of the Epilepsies in Adults and Children in Primary and Secondary Care. http://www.nice.org.uk/ guidance/cg137/resources/guidance-the-epilepsies-the-diagnosis-andmanagement-of-the-epilepsies-in-adults-and-children-in-primary-andsecondary-care-pdf, accessed in October 2013.

7. Dilorio C, Faherty B, Manteuffel B. The development and testing of an instrument to measure self-efficacy in individuals with epilepsy. J Neurosci Nurs. 1992;24:9-13

8. Ridsdale L, Virdi C, Noble A, Morgan A. Explanations given by people with epilepsy for using emergency medical services: a qualitative study. Epilepsy Behav. 2012;25:529-33.

9. Gillett M, Dallosso HM, Dixon S, Brennan A, Carey ME, Campbell MJ, et al. Delivering the diabetes education and self management for ongoing and newly diagnosed (DESMOND) programme for people with newly diagnosed type 2 diabetes: cost effectiveness analysis. BMJ. 2010;341:c4093. doi:10.1136/bmj.c4093.

10. Jain P, Patterson VH, Morrow Jl. What people with epilepsy want from a hospital clinic. Seizure. 1993;2:75-8

11. Bradley PM, Lindsay B. Care delivery and self-management strategies for adults with epilepsy. Cochrane Database Syst Rev. 2008;1, CD006244. doi:10.1002/14651858.CD006244.pub2.

12. Ramaratnam S, Baker GA, Goldstein LH. Psychological treatments for epilepsy. Cochrane Database Syst Rev. 2008;3, CD002029. doi:10.1002/ 14651858.CD002029.pub3.

13. Ried S, Specht U, Thorbecke R, Goecke K, Wohlfarth R. MOSES: an educational programme for patients with epilepsy and their relatives. Curr Prob E. 2001;16:147-54

14. Kralj-Hans I, Goldstein LH, Noble AJ, Landau S, Magill N, McCrone P, et al Self-Management education for adults with poorly controlled eplLEpsy (SMILE (UK)): a randomised controlled trial protocol. BMC Neurol. 2014;14:69. doi:10.1186/1471-2377-14-69.

15. Cramer JA, Perrine K, Devinsky O, Bryant-Comstock L, Meador K, Hermann B. Development and cross-cultural translations of a 31-item quality of life in epilepsy inventory. Epilepsia. 1998;39:81-8.

16. Baker GA, Jacoby A, Buck D, Stalgis C, Monnet D. Quality of life of people with epilepsy: a European study. Epilepsia. 1997;38:353-62.
17. Thapar A, Kerr M, Harold G. Stress, anxiety, depression, and epilepsy: investigating the relationship between psychological factors and seizures. Epilepsy Behav. 2009;14:134-40

18. Jacoby A, Baker G, Smith D, Dewey M, Chadwick D. Measuring the impact of epilepsy: the development of a novel scale. Epilepsy Res. 1993;16:83-8.

19. Zigmond AS, Snaith RP. The hospital anxiety and depression scale. Acta Psychiatr Scand. 1983;67:361-70.

20. Jacoby A. Felt versus enacted stigma: a concept revisited. Soc Sci Med 1994;38:269-74.

21. Wagner AK, Keller SD, Kosinski M, Baker GA, Jacoby A, Hsu MA, et al. Advances in methods for assessing the impact of epilepsy and antiepileptic drug therapy on patients' health-related quality of life. Qual Life Res. 1995:4:115-34

22. Cramer JA, Arrigo C, Van Hammee G, Gauer LJ, Cereghino JJ. Effect of levetiracetam on epilepsy-related quality of life. N132 Study Group. Epilepsia. 2000;41:868-74.

23. Kaminow L, Schimschock JR, Hammer AE, Vuong A. Lamotrigine monotherapy compared with carbamazepine, phenytoin, or valproate monotherapy in patients with epilepsy. Epilepsy Behav. 2003;4:659-66.

24. Campbell MK, Fayers PM, Grimshaw JM. Determinants of the intracluster correlation coefficient in cluster randomized trials: the case of implementation research. Clin Trials. 2005;2:99-107.

25. Little RJ, D'Agostino R, Cohen ML, Dickersin K, Emerson SS, Farrar JT, et al. The prevention and treatment of missing data in clinical trials. N Engl J Med. 2012;367:1355-60. doi:10.1056/NEJMsr1203730.

26. Schulz KF, Altman DG, Moher D, CONSORT Group. CONSORT 2010 Statement: updated guidelines for reporting parallel group randomised trials. Trials. 2010;11:32. doi:10.1186/1745-6215-11-32.

27. Zwarenstein M, Treweek S, Gagnier JJ, Altman DG, Tunis S, Haynes B, et al. Improving the reporting of pragmatic trials: an extension of the CONSORT statement. BMJ. 2008;337:a2390. doi:10.1136/bmj.a2390.

28. Boutron I, Moher D, Altman DG, Schulz KF, Ravaud P, CONSORT Group. Extending the CONSORT statement to randomized trials of nonpharmacologic treatment: explanation and elaboration. Ann Intern Med. 2008;148:295-309.

29. Sterne JAC, White IR, Carlin JB, Spratt M, Royston P, Kenward MG. Multiple imputation for missing data in epidemiological and clinical research: potential and pitfalls. Br Med J. 2009;339:B2393. doi:10.1136/Bmj.B2393.

30. Jackson $D$, White $\mathbb{I R}$, Leese M. How much can we learn about missing data?: an exploration of a clinical trial in psychiatry. J R Stat Soc Ser A Stat Soc 2010;173:593-612.

31. White IR, Thompson SG. Adjusting for partially missing baseline measurements in randomized trials. Stat Med. 2005;24:993-1007. doi:10.1002/sim.1981.

32. Angrist JD, Imbens GW, Rubin DB. Identification of causal effects using instrumental variables. J Am Stat Assoc. 1996:91:444-55. doi:10.2307/ 2291629.

33. Curtis L. Unit costs of health and social care. Canterbury: Personal Social Services Research Unit; 2010.

34. Lewin S, Glenton C, Oxman AD. Use of qualitative methods alongside randomised controlled trials of complex healthcare interventions: methodological study. BMJ. 2009;339:B3496. doi:10.1136/Bmj.B3496.

35. Blake RV, Wroe SJ, Breen EK, McCarthy RA. Accelerated forgetting in patients with epilepsy: evidence for an impairment in memory consolidation. Brain. 2000;123(Pt 3):472-83.

36. Green J, Thorogood N. Qualitative Methods for Health Research. New York: Sage: Sage Publications; 2004

37. Ritchie J, Spencer L, O'Connor W. Carrying out qualitative analysis. In: Ritchie J, Lewis J, editors. Qualitative Research Practice. New York: Sage: Sage Publications; 2003. p. 219-262. 